\title{
DISTRIBUTION OF PRESSURE OF GAS UNDER ACTION OF CENTRIFUGAL FORCES
}

\author{
Krokhmal V.V. \\ The Institute of Metallurgy, Mechanical Engineering and \\ Transport \\ Peter the Great Saint-Petersburg polytechnic university \\ Saint-Petersburg, Russia \\ w.krohmal@gmail.com
}

\author{
Klyukin V.Yu. \\ The Institute of Metallurgy, Mechanical Engineering and \\ Transport \\ Peter the Great Saint-Petersburg polytechnic university \\ Saint-Petersburg, Russia \\ kljukin@mail.ru
}

\author{
Sorokina E.V. \\ The Institute of Metallurgy, Mechanical Engineering and Transport \\ Peter the Great Saint-Petersburg polytechnic university \\ Saint-Petersburg, Russia \\ cspjn@mail.ru
}

\begin{abstract}
The pressure distribution of compressed air inside a closed cylinder under the influence of centrifugal forces is reproduced by rotating the centrifuge rotor. It is shown that during rotation, the centrifugal acceleration increases more intensively than the change in the gas pressure in a closed vessel. It is shown that in some areas of closed vessels placed on the rotating rotor of a centrifuge, vacuum may occur. The dependence of the gas pressure distribution on the rotor length of the test centrifuge is obtained. Dependences of the pressures at the end faces of the cylinder under load on the pressure of the initially injected gas, as well as the dependence of these pressures on the rotational speed, are constructed.
\end{abstract}

Keywords - centrifuge, cylinder, gas, gas distribution, centrifugal force

\section{INTRODUCTION}

Testing is an integral part in designing and creating any new technical objects, instruments, etc. To date, test studies are conducted in all industries. These tests are part of the life cycle of the creation of most new objects and products. In carrying out such a study, objects are subjected to loads that are comparable or exceed the loads in real conditions. The purpose of the testing of objects is to find out the response of the object to specific conditions and limit values of the load. Also, carrying out of test studies is economically advantageous for enterprises and plants from the point of view that they make it possible to identify possible errors in the creation of objects and thereby prevent the production of large quantities of defective products [1]. This is especially advantageous for the serial production of products.

For the implementation of test studies to date, test stands are used. Test stand - laboratory equipment, which is intended for special, control tests of various objects. After the research of the objects on the test bench, tests are carried out in real conditions. The advantage of tests on the test bench before the tests in real conditions is also to identify any design flaws in the objects created, which is also economically advantageous.
There are different types of stands with a fairly extensive list of tests conducted. Different loads on the object are realized at the stands, such as mechanical impacts (acceleration, speed, vibration, impact loads, tensile stress, compression, bending, etc.), climatic influences (temperature, humidity, atmospheric pressure, fog, dew, wind loads, dust, various kinds of radiation). Assess reliability (durability, stability, maintainability), endurance (fatigue of materials) of objects.

There are also stands that perform complex tests of objects where, for example, mechanical and climatic effects are carried out simultaneously. The advantage of such stands is the implementation of several tests on one stand, which is much more advantageous than carrying out each test separately on a separate test bench. However, such stands are most often complex in their design, so few are produced.

In this paper, the object of investigation is a test bench - a centrifuge, on the rotor of which a mechanical vibrator with a pneumatic shock absorber is fixed. At this stand, it is possible to perform complex tests of objects for acceleration and, at the same time, for vibrational forces that coincide in direction with centrifugal force. Such tests are necessary for facilities that are widely used in the aviation, military and space industries, since such objects in use are subjected to similar loads that reproduce the test benches, which is quite relevant in the industry today [2].

\section{INVESTIGATION OF GAS PRESSURE DISTRIBUTION IN A CLOSED CYLINDER}

In the study of the operation of a pneumatic shock absorber with a mechanical vibrator, which is fixed on the rotor of a test centrifuge (Fig. 1), it is necessary to conduct a study of the behavior of air in the field of centrifugal forces. To facilitate the solution of the task, instead of a pneumatic shock absorber, a closed hollow cylinder is used as the basis, which is installed at the end of the rotor of the test centrifuge. Figure 1 shows the structural and functional design of the 
centrifuge-based test bench, which gives the most detailed picture of the test stand as a whole [3].

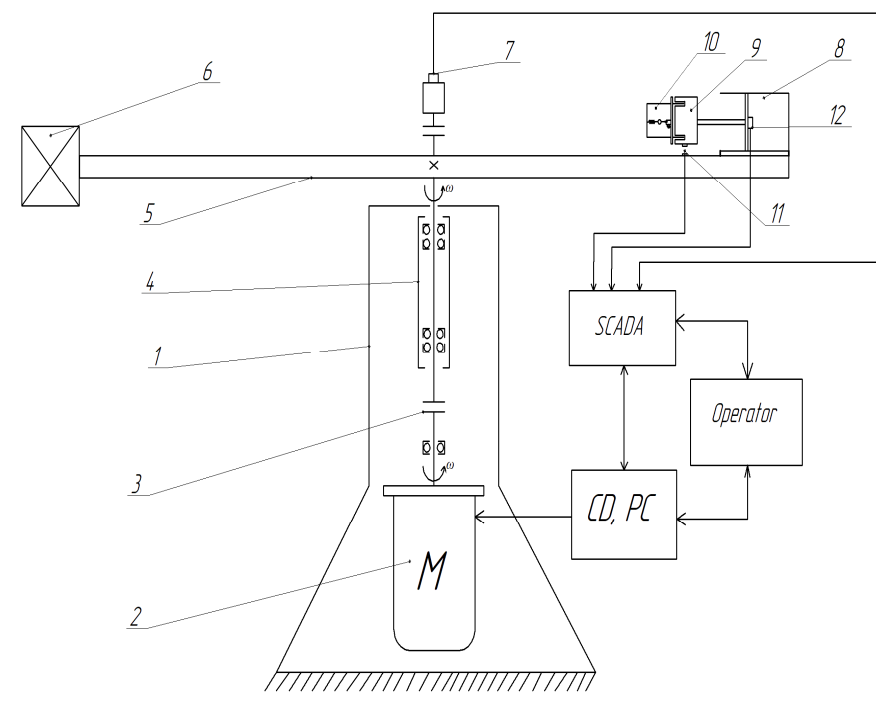

Fig. 1. Structural and functional scheme of a centrifuge with a mechanical vibrator and a pneumatic shock absorber on the rotor.

The following elements are included in the structurefunctional scheme: 1 - supporting structure, 2 - motor, 3 coupling, 4 - spindle, 5 - rotating platform (rotor), 6 counterweight, 7 - speed sensor, 8 - pneumatic shock absorber, 9 - mechanical vibrator, 10 - test object (in this case an accelerometer), 11, 13 - position sensor, 12 - pressure sensor. When the engine is running, rotation is transferred to the rotor, the speed sensor locks the speed sensor. At a constant rotor speed, a mechanical vibrator is turned on. The vibration is transmitted to the accelerometer. Coupled with a mechanical vibrator, a pneumatic shock absorber limits the vibrations transmitted to the rotor itself. Displacement of the piston of the pneumatic shock absorber, connected with the mechanical vibrator, fix the position sensors. The pressure level under the damper piston is measured by a pressure sensor. Information from the sensors is transferred to the SCADA system. In its turn SCADA - system - software and hardware complex for data collection and dispatch control. The received information is analyzed by the operator and the control device, which in turn controls the centrifuge, based on the data received from the sensors [4].

In order to begin the study of the operation of the pneumatic shock absorber together with the mechanical vibrator, which are installed on the rotor of the centrifuge of the test bench, it is necessary to analyze the behavior of the gas in the closed chamber of the cylinder during rotation of the centrifuge. For analysis, it is necessary to determine the gas pressure at each point of the chamber along the length of the cylinder when the centrifuge rotor rotates [5].

The diagram with the distribution of forces acting inside the cylinder is shown in figure 2 [6].

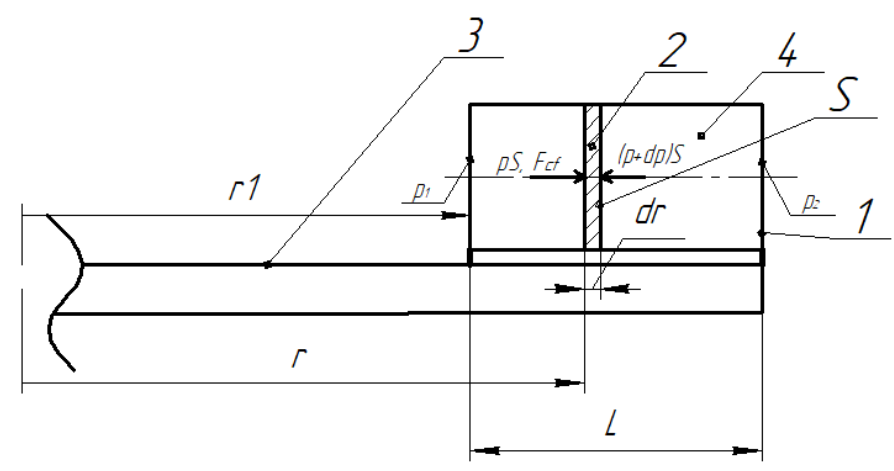

Fig. 2. Schematic of a part of centrifuge with a closed cylinder.

Figure 2 shows a schematic of a part of a centrifuge with a closed cylinder 1 , which is rigidly fixed to the rotor 3 . The chamber of a closed cylinder filled with gas 4 is investigated. Let us isolate the elementary volume 2 , determined by the cross-sectional area of the cylinder and the length $d r$. The length of the rotor from the axis of rotation to the cylinder is $r_{1}=2,5 \mathrm{~m}$, the total length of the cylinder chamber is $L=0,5 \mathrm{~m}$.

Let us consider the elementary volume of gas in the chamber under investigation and subjected to acceleration caused by centrifugal forces.

Its volume is determined in accordance with formula (1):

$$
d V=S d r
$$

where $S$-the cross-sectional area of the chamber, $\mathrm{m}^{2} ; d r-$ length of the allocated elementary volume of gas in the chamber, $\mathrm{m}$.

The mass of gas in this elementary volume is calculated by the following:

$$
d m=\rho(p) d V=\rho(p) S d r
$$

where $\rho(p)$-the gas density, depending on the pressure, $\frac{\mathrm{kg}}{\mathrm{m}^{2}}$.

The air density is determined from the ClapeyronMendeleev equation (assuming that the gas is dry):

$$
\frac{p V}{T}=\frac{m}{M} R
$$

where $T$ - the absolute temperature of the gas, $\mathrm{K} ; M-$ molar mass of gas (equal to air $29 * 10^{-3} \frac{\mathrm{kg}}{\mathrm{mol}}$ ) $; R$ - the universal gas constant (equal to $8.314 \frac{\mathrm{J}}{\mathrm{kg} \& \mathrm{~K}}$ ), $\mathrm{V}$ - the volume of gas, $\mathrm{m}^{3} ; m$ weight of gas, $\mathrm{kg} ; p$ - gas pressure, $\mathrm{Pa}$.

Since the density of the gas is $\rho=\frac{m}{V}$, then $\rho(p)=p \frac{M}{R T}$. 
In accordance with formula (2), we obtain the dependence for determining the mass of the gas in the allocated elementary volume:

$$
d m=p \frac{M}{R T} S d r
$$

The centrifugal force $d F_{\text {uø }}$, acting on the selected volume of gas, is determined in accordance with the following formula:

$$
d F_{c f}=\omega^{2} r d m
$$

where $\omega-$ angular rotational speed of the rotor of the test centrifuge, $\frac{\mathrm{rad}}{\mathrm{s}} ; r$ - the distance from the axis of rotation of the centrifuge to the elementary volume of the gas, $m$.

Under the assumption of a static load acting on the gas of a given volume, we obtain the equilibrium equation for the elementary volume of the gas:

$$
d F_{c f}+p S-S(p+d p)=0
$$

where $d p$-the increment of the gas pressure at a distance $d r$ from the axis of rotation, $\mathrm{Pa}$.

Substituting relationships (5) and (4) into equation (6), we obtain:

$$
\omega^{2} r p \frac{M}{R T} S d r-S d p=0
$$

This implies:

$$
\frac{d p}{p}=\omega^{2} \frac{M}{R T} r d r
$$

This dependence allows us to find the gas pressure distribution within the chamber as a function of the distance from the axis of rotation. We assume that the gas pressure at a distance $r_{1}$ from the rotation axis will be $p_{1}$.

Then the gas pressure at an arbitrary point of the cylinder located on the rotating centrifuge will be determined from the expression:

$$
\int_{p_{1}}^{p} \frac{d p}{p}=\int_{r_{1}}^{r} \omega^{2} \frac{M}{R T} r d r
$$

Integrating, we obtain:

$$
\ln \left(\frac{p}{p_{1}}\right)=\omega^{2} \frac{M}{R T} \frac{r^{2}-r_{1}^{2}}{2}+\mathrm{C}
$$

where $\mathrm{C}$ - the integration constant.

The value of $\mathrm{C}$ is determined from the condition that the gas pressure at a distance $r_{1}$ from the rotation axis will be $p_{1}$ :

$$
\mathrm{C}=\ln (1)=0
$$

Thus, we obtain an expression for determining the distribution of the gas pressure in the cylinder cavity:

$$
p=p_{1} e^{\frac{\omega^{2} M\left(r^{2}-r_{1}^{2}\right)}{2 R T}}=p_{1} E_{z}
$$

depending on the distance from the axis of rotation:

where $E-$ a dimensionless parameter that determines the change in the gas pressure along the length.

In accordance with expression (12), it is possible to construct the pressure distribution in the cylinder chamber with a change in the distance from the rotation axis and the rotation speed of the centrifuge. The result is shown in figure 3 [7].

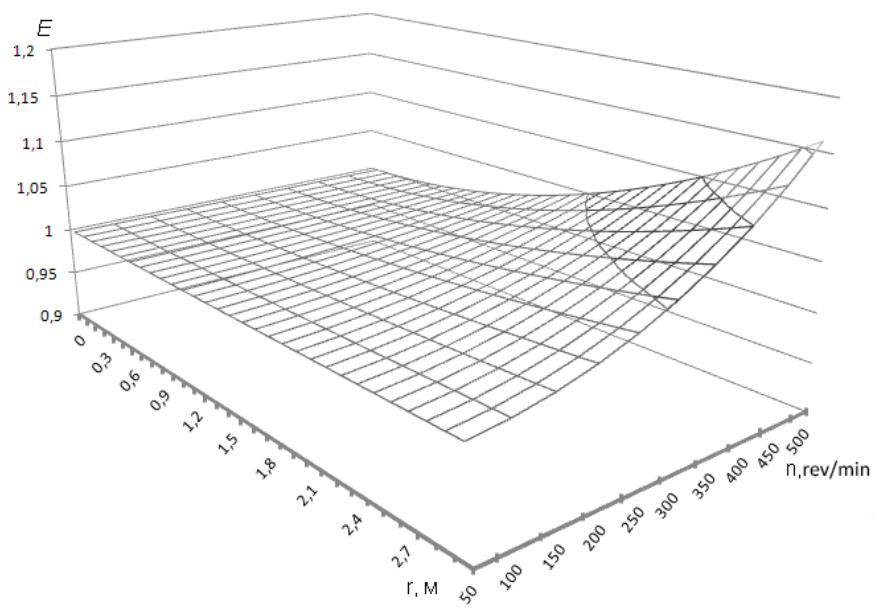

Fig. 3. Parameter value from the rotation speed of the rotor of the centrifuge and the radius from the axis of rotation.

The graph in figure 3 shows that, under certain conditions, the gas is redistributed within the closed volume under the action of centrifugal forces. This changes the gas pressure depending on the distance to the axis of rotation. Under the influence of centrifugal forces there is a redistribution of gas inside a closed chamber. The degree of redistribution depends on the rotational speed of the centrifuge and the distance from the axis of rotation. Gas redistribution leads to a change in the gas pressure along the length of the chamber. 
From the obtained data, it is possible to calculate the initial pressure of the gas filling the cylinder before the rotation begins, that is, the gas pressure in the cylinder chamber without a centrifugal load (without rotating the rotor of the test centrifuge). The gas pressure in the chamber in the absence of a load will be the same along the length of the chamber. The pressure graph from the cylinder length, in this case, looks like a horizontal straight line, the area of the graph under which is equivalent to the energy of the gas. This energy is preserved even after redistribution of the gas pressure inside the cylinder. Thus, the areas under the graphs should be the same. The area under the linear graph of the gas pressure distribution without load will be: $S=a * r$, where $r$ - the cylinder chamber length (rectangle width), $a-$ the equivalent of the initial pressure $\mathrm{p}_{n}$ (rectangle height):

$h=\frac{r}{n} ; \quad S=h\left(\Sigma p-\frac{\left(p_{1}+p_{2}\right)}{2}\right)(13)$,

where $h$ - step, $r$ - the length of the cylinder chamber, $n-$ the number of discrete sections, $S$ - the area under the pressure distribution graph, $\Sigma p$ - the sum of all pressure values in each section of the chamber, $p_{1}$ and $p_{2}-$ the pressure at one and the second end of the chamber, respectively.

Given that the areas under the pressure distribution diagrams with and without load should be equal, let us calculate the area under the pressure distribution graph under load. The calculation is made by the method of trapezium, according to which the area formula is as follows:

Considering the area found under the pressure distribution graph under load, it is possible to find the initial pressure in the chamber of the closed cylinder without load according to the following formula:

$$
p_{n}=\frac{s}{r}
$$

where $p_{n}-$ the initial pressure in the chamber without load, $S$ - the area under the pressure distribution diagram with the load, $r$ - the length of the cylinder chamber.

Figure 4 shows the dependence of the gas pressure distribution on the rotor speed of the centrifuge at an initial pressure of 1 bar.

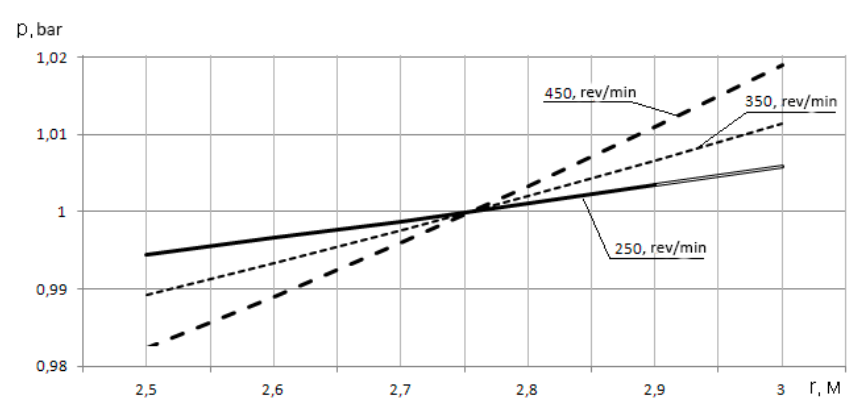

Fig. 4. Gas pressure distributions inside a closed vessel of the cylindrical radial arrangement with the initial pressure of 1 bar.
Figure 4 shows the distribution of the gas pressure initially injected at an absolute pressure of 1 bar into a closed vessel of cylindrical shape. One of the end walls of the vessel is located at a distance of $2.5 \mathrm{~m}$ from the axis of rotation of the rotor, and the second - at a distance of $3 \mathrm{~m}$.

Inside the vessel, under the action of centrifugal forces, a redistribution of the gas takes place, which leads to the appearance of rarefaction in one part of the vessel. The higher the rotor speed of the centrifuge rotor, the higher the difference between the pressure at the end walls of the vessel, respectively.

Let us construct the dependence of the centripetal acceleration on the rotation speed of the centrifuge rotor and the distance to the axis of rotation. The result of the dependence is shown in figure 5 [8].

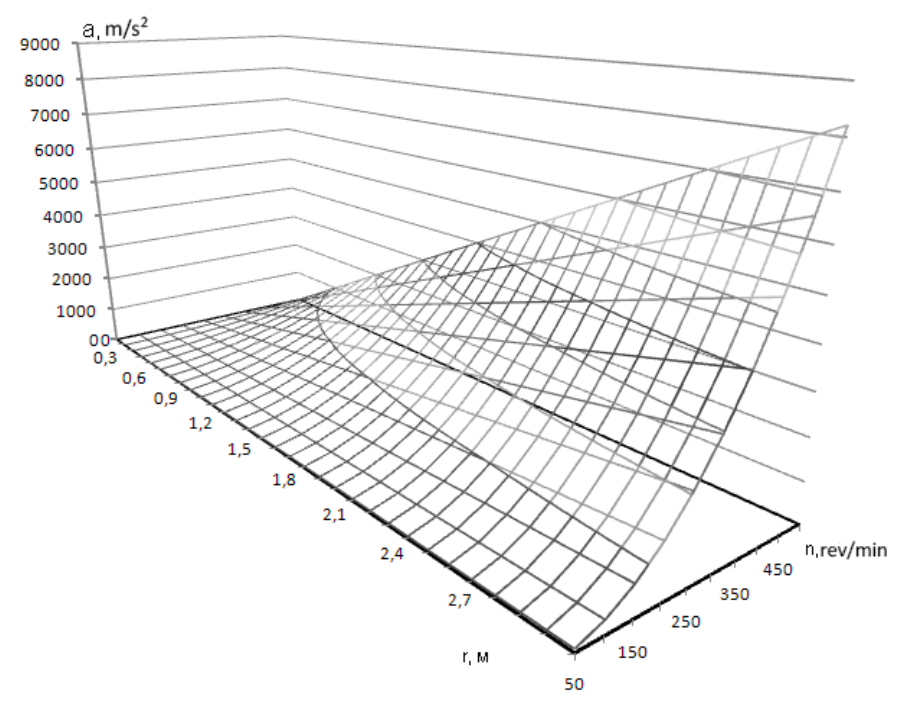

Fig. 5. The value of the centripetal acceleration $a$ from the rotational speed of the centrifuge $n$ and the radius from the axis of rotation $r$.

Figure 5 shows the increase in the centripetal acceleration with increasing rotational speed of the centrifuge rotor and the distance from the axis of rotation.

MatLab software package was used for further calculations and constructing pressure distribution graphs in the vessel. It is a package of applied programs for solving technical computing problems and the programming language of the same name used in this package. The program is presented in Appendix A to the thesis.

Before starting the program, you must set the necessary pressure at one end of the camera. Depending on the value of this pressure, the program determines the pressure at each subsequent section with a constant step.

In this case, the following values of the variables are given. The rotor speed of the test centrifuge is constant, is $n=$ 500 revolutions per minute ( $\omega=52,36$ radians per second). The length of the rotor of the test centrifuge, $r_{1}=2,5$ meters, the step between the discrete sections, $L=0,5$ meters, the step between the discrete sections, $l=0,05$ meters, the pressure at 
the starting point (at the first end of the chamber of the closed cylinder), $P=200000 \mathrm{~Pa}$ (2 bar). After the program was started, a graph was constructed of the pressure distribution in the chamber of the closed cylinder when the test centrifuge was rotated (figure 6) [9].

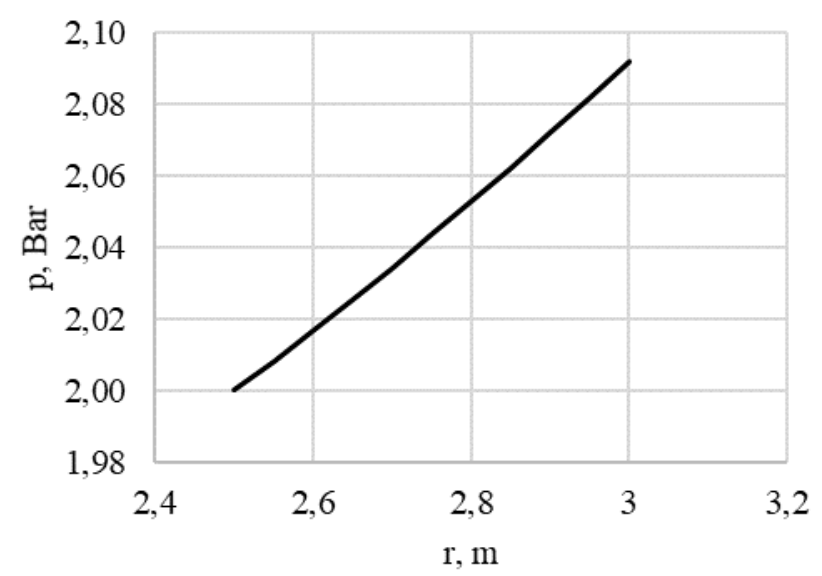

Fig. 6. Graph of the distribution of pressure in the cylinder chamber when the test centrifuge rotates.

Similarly, the pressure distribution can change when the cylinder position changes along the length of the centrifuge rotor. For lengths of 2, 2.5 and 3 meters, the pressure distributions are shown in figure 7.

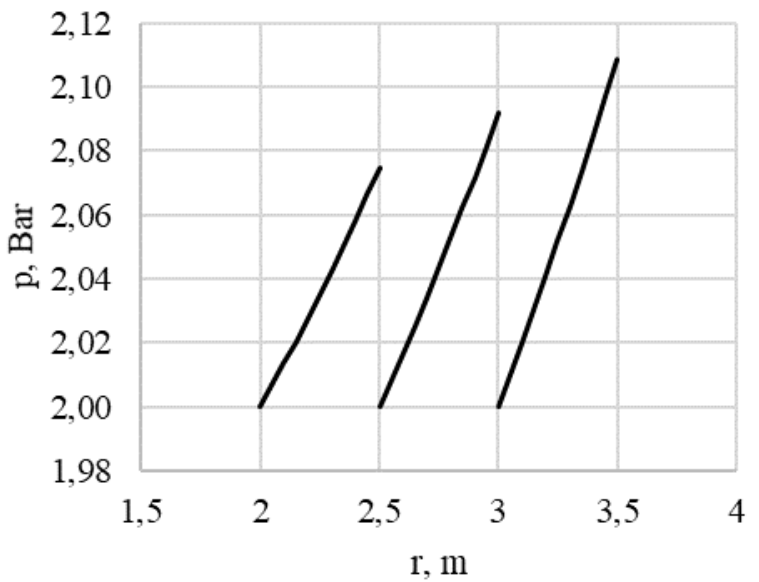

Fig. 7. Dependence of the distribution of pressure on the position of the cylinder on the rotor of the test centrifuge

It can be seen from figure 7 that the pressure at the righthand point $p_{2}$ of the cylinder chamber also increases along the length of the centrifuge rotor. This is due to the increase in centrifugal force. The same picture will be if you increase the length of the cylinder chamber.

Let us construct the dependences of the initial pressure $p_{n}$ on the pressures at the first and second ends of the cylinder chamber $p_{1}$ and $p_{2}$. The results of the calculations are given in Table 1, and the graphs are shown in figure 8.
TABLE I. INDICATORS $p_{n}$ OF VALUES $p_{1}$ AND $p_{2}$

\begin{tabular}{|c|c|c|}
\hline$p 1, \mathrm{~Pa}$ & $p 2, \mathrm{~Pa}$ & $p_{n}, \mathrm{~Pa}$ \\
\hline 50000 & 52263 & 51089 \\
\hline 100000 & 104530 & 102180 \\
\hline 150000 & 156790 & 153270 \\
\hline 200000 & 209050 & 204360 \\
\hline 250000 & 261310 & 255450 \\
\hline 300000 & 313580 & 306540 \\
\hline
\end{tabular}

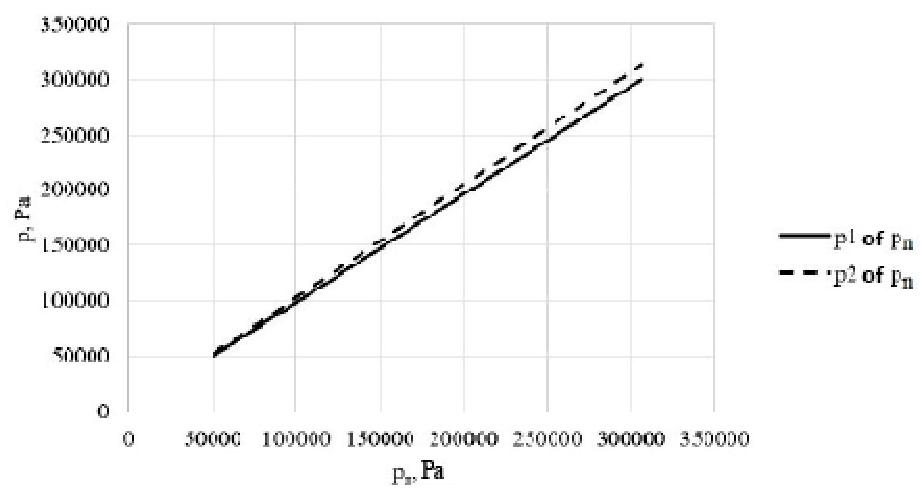

Fig. 8. Pressure dependencies $p_{1}$ and $p_{2}$ under load from the initial pressure $p_{n}$ without load.

The pressure dependence is linear.

Let us construct the dependences of the rotor speed on the initial pressure $p_{n}$ and pressure at the second end of the cylinder chamber $p_{2}$ with the load and the specified pressure at the first end of the chamber $-p_{1}=1$ bar. The results of the calculations are shown in Table 2 and the graphs are shown in figure 9 .

TABLE II. DEPENDENCE OF SPEED ROTATION VELOCITY OF

VALUES $p_{2}$ AND $p_{n}$

\begin{tabular}{|c|c|c|}
\hline $\mathrm{n}, \mathrm{rev} / \mathrm{min}$ & $p_{2}, \mathrm{~Pa}$ & $p_{n}, \mathrm{~Pa}$ \\
\hline 300 & 101630 & 100790 \\
\hline 350 & 102220 & 101070 \\
\hline 400 & 102910 & 101410 \\
\hline 450 & 103700 & 101780 \\
\hline 500 & 104530 & 102180 \\
\hline
\end{tabular}




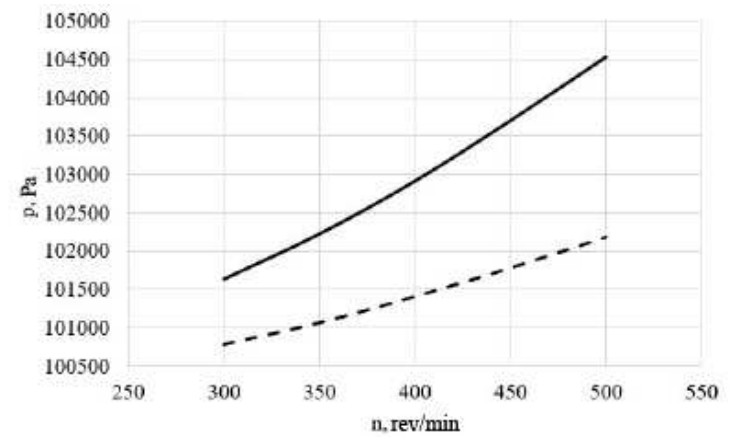

Fig. 9. Dependence of rotor speed of the centrifuge rotor on the initial pressure $p_{n}$ and pressure $\beta_{2}$ at the load.

As the rotation speed of the rotor increases, the centrifuges of pressure increase; this is due to the increase in the centrifugal force [10].

\section{CONCLUSIONS}

1. The article studies the distribution of compressed air inside a closed cylinder under the influence of centrifugal forces, which are reproduced by rotating the centrifuge rotor.

2. The dependences shown in figures 3, 4, and 5 show that, during rotation, the centrifugal acceleration increases more intensively than the gas pressure changes in a closed vessel.

3. It should also be noted that in some areas of closed vessels placed on the rotating rotor of a centrifuge, a vacuum may occur.

4. The dependence of the gas pressure distribution on the rotor length of the test centrifuge is obtained. Dependences of the pressures at the end faces of the cylinder under load on the pressure of the initially injected gas, as well as the dependence of these pressures on the rotational speed, are constructed.

\section{References}

[1] A.N. Popov, M.N. Polishchuk, Al. N. Timofeev, Stands for mechanical testing: textbook, SPb: Publishing house of Polytechnic. University, 2016.

[2] I.B. Chelpanov, M.I. Evstifeev, A.V. Kochetkov, "Rotary bench equipment for testing micromechanical devices", Internet - journal "Naukovedenie", vol. 5, pp.1-11, 2013.

[3] S.V. Pavlyuchenko and others, Schematics of technological machines. Rules for the implementation and synthesis of kinematic, pneumatic, hydraulic and electrical circuits: textbook, $\mathrm{SPb}$ : Publishing house Polytechnic. University, 2013.

[4] V.I. Karazin, D.P. Kozlikin, A.A. Sukhanov, I.O. Khlebosolov, "One stable scheme of centrifugal forces dynamic balance", Lecture notes in mechanical engineering, pp. 75-85, 2016.

[5] P.A. Andrienko, D.P. Kozlikin, A.V. Hisamov, I.O. Hlebosolov, "About the using of non-contact measurement systems in centrifugal srands. Modern mechanical engineering", Science and Education: a scientific edition of the MSTU. N.E. Bauman, vol 3, p. 623, 2013.

[6] V.I. Karazin, D.P. Kozlikin, A.V. Hisamov "Peculiarities of calculation of the centrifugh rotor deformations", Modern mechanical engineering. Science and Education, vol. 3, pp. 766-778, 2013.

[7] V.I. Karazin, S.V. Kolesnikov, S.D. Litvinov, A.A. Sukhanov, I.O. Khlebosolov, "Peculiarities of simulation and reproduction of viberous exposure", Theory of mechanisms and machines, vol. 22, pp.55-64, 2013.

[8] V.I. Karazin, D.P. Kozlikin, A.A. Sukhanov, V.A. Tereshin, I.O Khlebosolov, "Some methods of sustainable equilibrium on relative moves at centrifugals", Modern mechanical engineering. Science and Education vol. 5, pp. 216-229, 2016.

[9] V.V. Krokhmal, Research of the pneumatic shock-absorber set on a rotor of the test centrifuge: thesis, master: 15.04.04, SPbPU, p. 103, 2017.

[10] V. Yu. Klyukin, D.P. Kozlikin, V.V. Krokhmal "Research of work of the pneumatic «spring» of vibrofug" Week of Science SPbPU: materials of a scientific conference with international participation. Institute of Metallurgy, Mechanical Engineering and Transport, part 2, pp. 9-11, 2017. 